\title{
Metodologia para racionalização do uso de energia elétrica para obtenção de força motriz em fábrica de ração: Estudo de caso
}

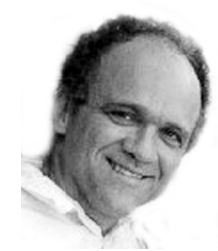

Delly Oliveira Filho ${ }^{1}$, Carlos A. Teixeira ${ }^{2}$, Adílio F. de Lacerda Filho ${ }^{3} \&$ José H. Martins ${ }^{4}$

1 DEA/UFV. CEP 36571-000, Viçosa, MG. Fone: (31) 3899-1897. E-mail: delly@ufv.br (Foto)

2 DEA/UFV. Fone: (31) 3899-1930. E-mail: carlos@vicosa.ufv.br

3 DEA/UFV. Fone: (31) 3899-1872. E-mail: alacerda@ufv.br

4 DEA/UFV. Fone: (31) 3899-1896. E-mail: jmartins@ufv.br

Protocolo 158 - 29/10/2002 - Aprovado em 27/1/2004

\begin{abstract}
Resumo: No cenário energético atual, a racionalização do uso de energia elétrica é uma ferramenta de apoio imprescindível para o crescimento econômico. No Brasil, as rações de suínos e aves são formuladas, basicamente, a partir de milho e farelo de soja, que, em geral, necessitam ser moídos para que a ração tenha uma granulometria específica. Com o objetivo de racionalizar o uso da energia elétrica, realizou-se um estudo de adequação de força motriz dos equipamentos em funcionamento, da fábrica de ração da Universidade Federal de Viçosa - UFV. Fez-se a avaliação do índice de carregamento e do rendimento dos motores, para a condição de carga de serviço. Para adequação do uso de força motriz, consideraram-se substituições dos motores elétricos que estavam acoplados aos equipamentos, por motores de alto rendimento adequados e/ou com o número de horas de funcionamento anual maximizado. Com a adoção da metodologia, o potencial total estimado de economia com energia elétrica anual seria de 22,60\%, (R\$1.728,28 em outubro de 2002), caso fossem realizados o aumento do número de horas de funcionamento anual e a adequação de força motriz com o uso de motores de alto rendimento, adequando-se também ás exigências eletromecânicas do moinho.
\end{abstract}

Palavras-chave: gerenciamento do lado da demanda, motor elétrico, economia de energia elétrica

\section{Methodology for matching motors for demand in animal feed plant: A case study}

\begin{abstract}
In the current energy scenario the management of electricity use is an indispensable support tool for the sustainable growth. In Brazil, the feed for swine and poultry are formulated, basically, with corn and bran of soy that usually need to be processed so that the feed has a specific size. This paper presents the management of the electricity use, by matching the use of motive force of animal feed factory at the Federal University of Viçosa. The evaluations of the motors' load and performance have been made at full load condition. For matching the use of motive force, substitutions of the electric motors by adequate motors of high performance and/ or by changing the number of hours of annual operation were considered. The methodology adopted presented a potential economy with energy of $22.60 \%, R \$ 1,728.28$ in October, 2002 , if the number of hours of annual operation increases, the adaptation of motive force with the use of motors of high performance, as well as sizing properly the mechanical demands of the factory, especially the mill were adopted.
\end{abstract}

Keywords: demand side management, electric motor, economy of electric energy

\section{INTRODUÇÃO}

A industrialização e o crescimento econômico, associado às crescentes inovações tecnológicas dos últimos anos, vêm causando aumento substancial na demanda de energia elétrica. A falta de investimentos no setor visando à geração, transmissão e distribuição de energia, faz da racionalização do uso de energia elétrica uma ferramenta de apoio imprescindível ao crescimento sustentável do País.
O planejamento energético tradicional procura expandir os recursos da oferta de energia elétrica com o propósito de atender, com segurança, principalmente os critérios de crescimento de demanda futura e minimizar o custo da expansão. Esses critérios de planejamento tradicional, aliados ao baixo custo de energia praticado até a década de 70 , levaram à estratégia, quase universal, da rápida expansão da capacidade geradora, sem uma preocupação explícita com o comportamento da demanda, dando pouca ênfase à eficiência do uso da energia. 
Com a crise do petróleo em 1973, o planejamento energético passou a contemplar opções de gerenciamento do lado da demanda (GLD) em virtude do alto custo da energia. O planejamento energético passou a ser integrado, englobando os recursos energéticos e o uso final da energia. Este modelo tem sido denominado Planejamento Integrado de Recursos (PIR). O PIR combina opções de tecnologia de oferta de eletricidade e melhoria da eficiência energética, para prover serviços de energia elétrica com menor custo, incluindo a contabilização dos custos social e ambiental. Segundo Jannuzzi \& Swisher (1997) o PIR inclui opções de GLD e do lado do suprimento (GLS).

O GLS visa racionalizar o uso da energia elétrica pelo lado do suprimento de energia, englobando medidas como a construção e ampliação de unidades geradoras, subestações e linhas de transmissão. O GLD atua diretamente na curva de carga de determinada concessionária de energia elétrica. Como exemplo de ações do GLD para diminuição da demanda de pico, citam-se: (i) o uso de equipamentos mais eficientes; (ii) o remanejamento de cargas; e (iii) o controle direto de cargas.

De acordo com Pinheiro (1986) a Eletrobrás estudou quatorze opções de GLD de energia elétrica, que incluem: (i) aumentar a eficiência de refrigeradores e ar condicionado; (ii) substituir lâmpadas incandescentes por fluorescentes comuns, ou compactas e incandescentes do tipo econômico; (iii) substituir lâmpadas de vapor de mercúrio por lâmpadas de vapor de sódio; (iv) substituir motores elétricos do tipo padrão por modelos de alto rendimento; e (v) adequação da força motriz. A adequação de força motriz foi a opção de menor custo, dentre as pesquisadas, ou seja, aquela cujo custo é inferior a 7\% de um centavo de real por $\mathrm{kWh}$ economizado.

Os principais usos finais da energia elétrica são o aquecimento, a iluminação e a força motriz. Segundo Low (1993) no Canadá a força motriz é responsável por cerca de $75 \%$ do uso final de energia elétrica utilizada no setor industrial.

No setor industrial brasileiro o uso de motores para força motriz representa, praticamente, metade do consumo de energia elétrica do País, aproximadamente $49 \%$ do consumo no setor (ELETROBRÁS, 1998).

Latorre et al. (1990) em estudos sobre o uso de força motriz nas indústrias brasileiras, abrangendo cerca de 50.000 motores, concluíram que cerca de $50 \%$ dos motores operavam com índice de carregamento abaixo de $80 \%$, ou seja, o percentual de energia mecânica demandada pela carga era, em 50\% dos casos, inferior ao recomendado. Neste caso, para motores com partida sem carga são recomendados índices de carregamento entre 80 a $100 \%$. Constatou-se, ainda, que cerca de $24,1 \%$ do total de motores analisados em nível nacional, estavam operando com $60 \%$ de carregamento, no máximo. Este estudo revelou que em alguns estados brasileiros 32,1\% dos motores operam com até $60 \%$ da carga. Este fato caracteriza o grande potencial de racionalização do uso de energia elétrica no setor industrial brasileiro. Esta racionalização do uso de força motriz poderá ser alcançada por meio de uma difusão maior do uso de motores de alta eficiência e um dimensionamento correto de motores elétricos, pela realização de testes e emissão de certificados e avaliação de desempenho de máquinas agrícolas.
Segundo Casp (1980) na criação de animais em escala industrial um dos fatores determinantes na maximização do lucro é, sem dúvida, a racionalização do uso de energia na produção de ração. Observa-se que a alimentação representa aproximadamente $75 \%$ do custo contínuo de um plantel, incluindo o custo de energia, enquanto $15 \%$ são custos relativos à depreciação e 10\% referentes à mão-de-obra.

No Brasil, as rações para suínos e aves são formuladas, basicamente, a partir de milho, farelo de soja e suplementos de minerais e vitaminas, sendo que o milho representa aproximadamente $75 \%$ da composição das rações para suínos e $65 \%$ das rações para aves. As rações para suínos e aves são produzidas na forma farelada, peletizada ou triturada, de acordo com a espécie, a idade, o estádio evolutivo do animal e/ou o manejo de alimentação adotado. Portanto, os ingredientes que participam da ração necessitam sofrer algum processo prévio de moagem (Zanotto et al., 1995).

É importante aumentar a taxa de conversão, isto é, converter o mínimo de ração no máximo de ganho de produção animal. A conversão alimentar é definida como sendo a razão entre o peso de alimento e a quantidade de produto convertida num intervalo de tempo (Casp, 1980).

Portanto, o estudo da racionalização do uso de energia é relevante em processos que utilizam a força motriz, como em instalações onde se processam as rações, utilizando-se motores elétricos. Em uma fábrica de ração existem vários motores em funcionamento, tais como os do sistema de moagem de granulados e do sistema de movimentação dos componentes, misturador e pré-misturador.

Dentre as opções de GLD, a racionalização do uso de força motriz tem sido relegada, em muitos casos, a segundo plano, por razões desconhecidas. Destaca-se, aqui, o fato de não se dispor de metodologias que sistematizem o uso de tração elétrica e, ainda, a não integração de programas computacionais de curvas características de motores elétricos, planilhas de cálculo e programas de digitalização de curvas. Como exemplo, cita-se a não integração dos programas de dimensionamento de bombas e ventiladores, com os de adequação de força motriz. Desta forma, este trabalho teve como objetivo propor uma metodologia para racionalização do uso de energia elétrica para a produção de força motriz e apresentar, como estudo de caso, uma análise da fábrica de ração da Universidade Federal de Viçosa.

\section{MATERIAL E MÉTODOS}

Este trabalho foi realizado no Laboratório de Energia e na fábrica de ração do Departamento de Engenharia Agrícola da Universidade Federal de Viçosa (UFV) em Viçosa, MG. Esta fábrica tem capacidade de $6,0 \mathrm{t} \mathrm{h}^{-1}$ e é aparelhada com os seguintes equipamentos:

a) sistema de moagem com moinho do tipo martelo

b) sistema de dosagem automática para cinco componentes de ração, sendo o restante dosado no pré-misturador

c) sistema de mistura, de ensaque, a granel e peletizado

d) transportadores de grãos, farelos e rações.

A Figura 1 mostra alguns equipamentos da fábrica de ração, ou seja, o sistema de transporte, o moinho principal, o misturador e o exaustor. 
A.

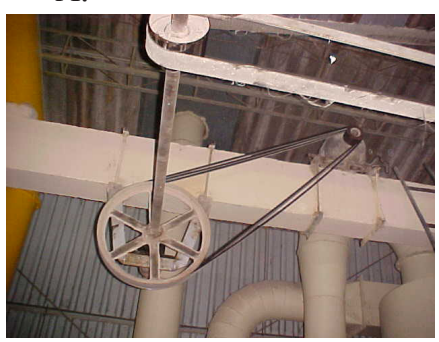

C.

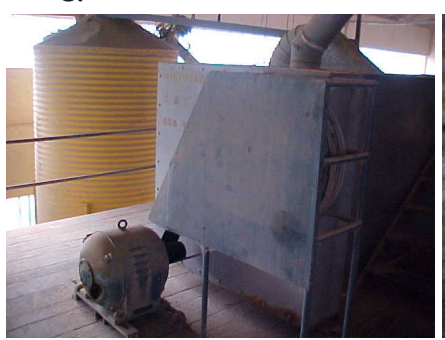

Figura 1. Vista de alguns equipamentos da fábrica de ração: (A) motor do sistema de transporte, (B) motor do moinho principal, (C) motor do misturador e (D) motor do exaustor

\section{Adequação de força motriz}

Para a adequação de força motriz é importante que se faça a avaliação do índice de carregamento e do rendimento do motor na condição de carga atual. O rendimento atual do motor elétrico pode ser determinado de forma direta ou indireta. De forma direta, é necessária a medição da rotação e do torque. A potência demandada é determinada pela Eq. 1:

$$
\mathrm{P}_{\mathrm{d}}=\mathrm{T}_{\mathrm{d}} \mathrm{W}
$$

em que:

$\mathrm{P}_{\mathrm{d}} \quad$ - potência demandada, $\mathrm{W}$

$\mathrm{T}_{\mathrm{d}} \quad$ - torque, $\mathrm{Nm}$

W - velocidade angular, $\operatorname{rad~s}^{-1}$

A velocidade angular é diretamente proporcional à rotação e pode ser expressa pela Eq. 2:

$$
\mathrm{P}_{\mathrm{d}}=\frac{2 \pi \mathrm{n} \mathrm{T}_{\mathrm{d}}}{60}
$$

em que:

$\begin{array}{ll}\mathrm{P}_{\mathrm{d}} & \text { - potência demandada, } \mathrm{W} \\ \mathrm{T}_{\mathrm{d}} & \text { - torque, } \mathrm{Nm}\end{array}$

A avaliação indireta do índice de carregamento pode ser feita de diversas formas: (i) pela medição da corrente elétrica do estator; (ii) pela medição do fator de potência e/ou (iii) pela medição do escorregamento.

A avaliação do índice de carregamento poderá ser feita também indiretamente, pela estimativa da carga mecânica. A determinação do rendimento por meio da medição da corrente elétrica é mais utilizada por ser a mais simples (Sá et al., 1990).

Para medição da corrente elétrica dos motores foi utilizado um amperímetro alicate. De posse das correntes elétricas do motor trabalhando com o carregamento de serviço diário e seus dados de placa, determinou-se o índice de carregamento (IC), que é a razão entre a potência real e a potência nominal, por meio da curva característica de corrente. Com o IC, determinouse o rendimento por meio da curva característica de rendimento (WEG, 2001). Na determinação do rendimento de motores elétricos, deve-se considerar, também, o desbalanceamento entre tensões, o nível de tensões e se o motor já foi rebobinado, haja vista a grande influência desses fatores no rendimento dos motores elétricos (ELETROBRÁS, 1998). Para considerar a influência do rebobinamento e/ou do reparo do motor em seu rendimento, deve-se utilizar um percentual de depreciação no rendimento do motor elétrico (Costa, et al., 1998). Neste trabalho, não foram levados em consideração os efeitos do rebobinamento nem de reparos do motor elétrico em seu rendimento (ELETROBRÁS, 1998).

As opções para adequação do uso de força motriz estudadas, foram:

a) substituição do motor padrão (PD) em uso, versus motor em uso, tipo padrão adequado à condição de carga

b) substituição do motor padrão (PD) em uso, versus motor novo de mesma potência de alto rendimento (AR)

c) substituição do motor padrão (PD) em uso, versus motor novo de alto rendimento (AR) adequado à condição de carga

d) aumento do número de horas de funcionamento anual.

Para cada situação são analisados os dados de entrada para a realização da análise econômica, tais como: número de horas anuais de funcionamento; vida útil; taxa de juros; aumento do preço da energia acima do índice da inflação, observada durante o horizonte de planejamento; preço do consumo e demanda e período do dia para efeito de cálculo de tarifas. A Figura 2 apresenta o fluxograma simplificado da metodologia para adequação de força motriz.

A Eq. 3 apresenta o potencial de economia com a melhoria de rendimento dos motores elétricos, em uma possível troca entre um motor 1 e um motor 2 :

$$
\Delta \eta=\frac{1}{\eta_{1}}-\frac{1}{\eta_{2}}
$$

em que:

$$
\begin{array}{ll}
\Delta \eta & \text { - economia de energia, } \% \\
\eta_{1} & \text { - rendimento do motor } 1, \%
\end{array}
$$$$
\eta_{2} \text { - rendimento do motor } 2, \%
$$

\section{Análise econômica}

$\mathrm{Na}$ análise econômica da racionalização do uso de força motriz utilizam-se parâmetros econômicos, como taxa interna de retorno (TIR), valor presente líquido (VPL), relação benefíciocusto (RBC) e taxa de retorno do capital (TRC). Para cada motor, os dados seguintes de entrada foram analisados para efeito da análise econômica: número de horas de funcionamento anual; vida útil; taxa de juros; aumento no preço da energia acima da inflação; horizonte de planejamento; preço do consumo e da demanda de energia elétrica, no horário de ponta e fora de ponta; preço dos motores e custo de manutenção. 


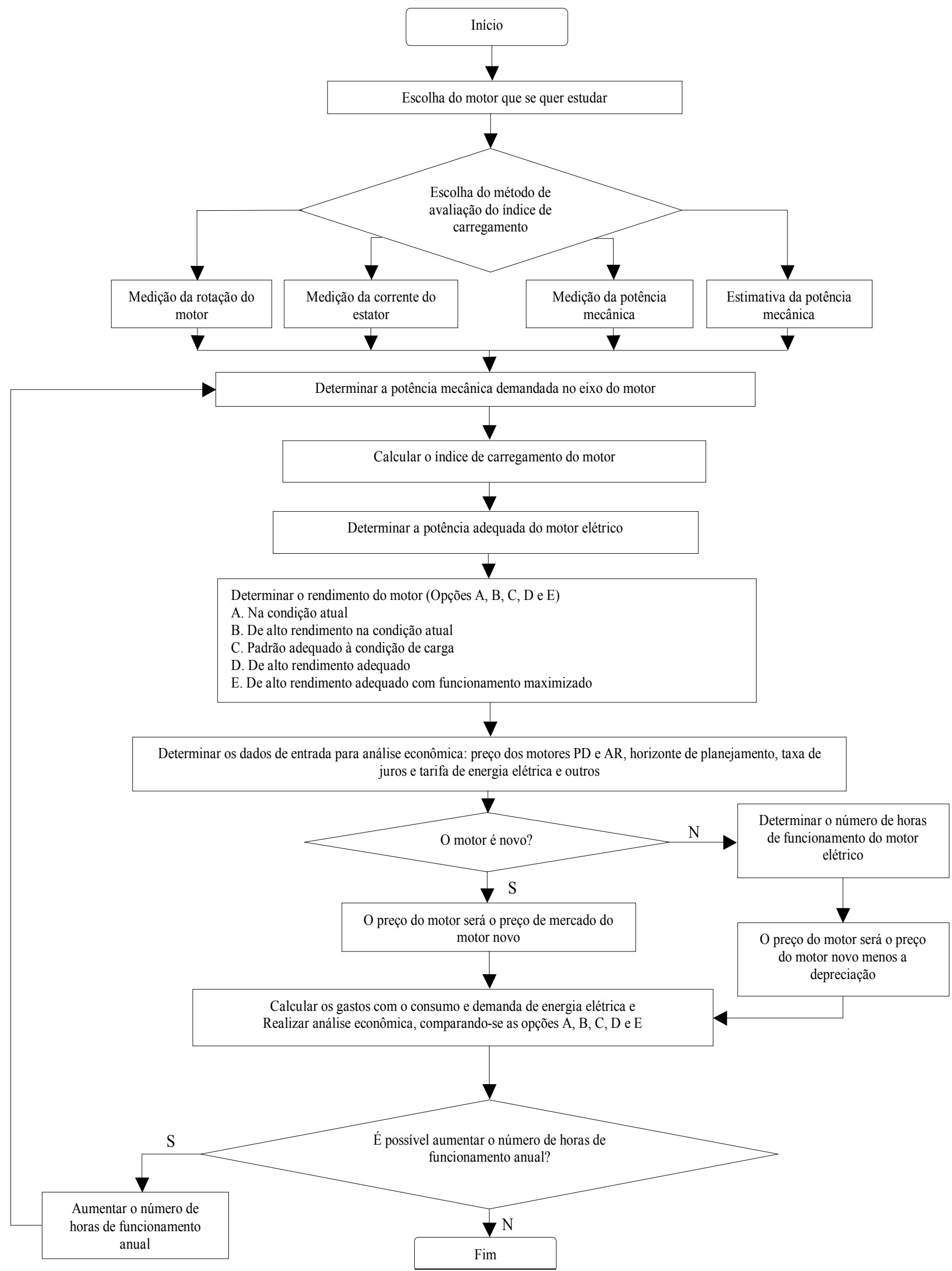

Figura 2. Fluxograma para adequação de força motriz 
O gasto com consumo de energia elétrica dos motores foi estimado empregando-se a Eq. 4:

$$
\mathrm{C}=\frac{0,736 \mathrm{P} \mathrm{IC} \mathrm{T}_{\mathrm{c}} \mathrm{h}}{\eta}
$$

em que:

$\mathrm{C}$ - gasto com consumo de energia elétrica, $\mathrm{R} \$ \mathrm{ano}^{-1}$

$\mathrm{P}$ - potência nominal do motor elétrico, $\mathrm{cV}$

0,736 - fator de conversão de cv para $\mathrm{kW}$

IC - índice de carregamento do motor elétrico, \%

$\mathrm{T}_{c} \quad$ - tarifa de consumo, $\mathrm{R} \$ \mathrm{kWh}^{-1}$

$\mathrm{h}$ - número de horas de funcionamento anual, $\mathrm{h}$

$\eta \quad$ - rendimento do motor elétrico, decimal

O gasto com demanda de energia elétrica dos motores foi estimado por meio da Eq. 5:

$$
\mathrm{D}=\frac{0,736 \mathrm{P} \mathrm{IC} \mathrm{T}_{\mathrm{d}} 12}{\eta}
$$

em que:

D - gasto com demanda de energia elétrica, $\mathrm{R} \$ \mathrm{ano}^{-1}$

$\mathrm{T}_{\mathrm{d}} \quad$ - tarifa de demanda, $\mathrm{R} \$ \mathrm{~kW}^{-1}$

12 - número de meses do ano

\section{RESULTADOS E DISCUSSÃO}

Seguem abaixo, como forma de exemplo da metodologia, quatro situações de troca de motores elétricos englobando o cálculo da potência e índice de carregamento dos motores adequados e, posteriormente, a aplicação da metodologia na fábrica de ração da UFV.

\section{Motor padrão versus motor padrão adequado à condição de carga em uso}

As Tabelas 1 e 2 exemplificam detalhadamente os passos (Calcular o índice de carregamento do motor e Determinar a potência adequada do motor elétrico) da Figura 2 para adequação de força motriz de um motor padrão em funcionamento, por outro padrão adequado à condição de carregamento, em uso.

$\mathrm{Na}$ escolha da potência mecânica requerida, a potência de $44,20 \mathrm{~kW}(60 \mathrm{cv})$ é o valor comercial da potência de motores. Neste caso, o motor a ser adotado seria um motor de potência igual a 44,20 kW (60 cv). Então, o IC do motor seria 100\%; aplicando-se a Eq. 3, observou-se perda de $0,36 \%$ no rendimento do sistema em relação ao uso do motor padrão, mas com 11,00 kW (15 cv) de potência mecânica requerida a menos; portanto, a diferença de custo entre os motores deve ser compensada pela diminuição da potência requerida. Este dado afeta também a corrente de partida do motor, que será consideravelmente menor.

Como a potência de $30,9 \mathrm{~kW}$ (42 cv) não é um valor comercial de potência de motores elétricos, deve-se escolher o valor mais próximo de potência acima da selecionada. Neste caso, o motor a ser selecionado é um motor com potência de $36,80 \mathrm{~kW}(50 \mathrm{cv})$. O IC será, neste caso, 84\% (42/50). Nesta substituição haverá um potencial de economia de $5 \%$ no rendimento do sistema, pela aplicação da Eq. 3 .
Tabela 1. Comparação entre a substituição de um motor padrão em uso por um motor padrão adequado à condição de carga em uso

\begin{tabular}{lc}
\hline \multicolumn{1}{c}{ Parâmetros Avaliados } & Valores \\
\hline Potência & $55,20 \mathrm{~kW}(75 \mathrm{cv})$ \\
Índice de carregamento & $80 \%$ \\
Rendimento & $91,5 \%$ \\
$\quad$ Resultados (motor - padrão adequado à condição de carga) \\
Potência & $55,2 \times 0,8=44,20 \mathrm{~kW}(60 \mathrm{cv})$ \\
Motor escolhido & $60 \mathrm{cv}$ \\
Índice de carregamento & $60 / 60=100 \%$ \\
Rendimento & $91,20 \%$ \\
\hline
\end{tabular}

Tabela 2. Comparação entre a substituição de um motor padrão em uso por um motor padrão adequado à condição de carga

\begin{tabular}{|c|c|}
\hline Parâmetros Avaliados & Resultados \\
\hline Potência do motor & $73,00 \mathrm{~kW}(100 \mathrm{cv})$ \\
\hline IC do motor & $42 \%$ \\
\hline Rendimento do motor & $87,50 \%$ \\
\hline \multicolumn{2}{|c|}{ Resultados } \\
\hline Potência demandada do motor & $73,60 \times 0,42=30,90 \mathrm{~kW}(42 \mathrm{cv})$ \\
\hline Potência do motor escolhido & $36,80 \mathrm{~kW}(50 \mathrm{cv})$ \\
\hline IC do motor escolhido & $42 / 50=84 \%$ \\
\hline Rendimento do motor & $91,5 \%$ \\
\hline
\end{tabular}
em uso

A análise é feita com base em parâmetros técnicos e econômicos, considerando-se a troca de um motor padrão em uso por um outro padrão também em uso, adequado à nova condição de carga e potência. Esta situação pode ocorrer quando há remanejamento de motores internamente, disponibilizando-se alguns motores do tipo padrão, que podem ser adequados em outras condições de uso. Com esta opção, o investimento em novos motores adequados será nulo ou reduzido.

O simples remanejamento interno dos motores pode realizar grande parte da adequação de força motriz de uma indústria com a utilização de seus próprios motores, com a vantagem de que agora estarão instalados os motores de potência adequada para as respectivas máquinas. Deve-se considerar que na compra de novos motores existem programas governamentais que dão subsídios para a aquisição de motores eficientes, como é o caso do programa de incentivo ao uso de motores de alto rendimento da CEMIG e do PROCEL da ELETROBRÁS. Com isso, o investimento em novos motores torna-se viável, na maioria das vezes.

\section{Motor padrão em uso versus motor novo de mesma potência de alto rendimento}

A análise baseia-se em parâmetros técnicos e econômicos para se efetuar a troca de um motor padrão em funcionamento, por um motor de alto rendimento novo, ambos de mesma potência e índice de carregamento. A Tabela 3 exemplifica detalhadamente os passos (Calcular o índice de carregamento do motor e Determinar a potência adequada do motor elétrico) da Figura 2, utilizada na adequação de força motriz de um motor padrão em funcionamento, por um motor de alto rendimento (AR) novo.

Nesta situação, como 55,20 kW (75 cv) é o valor comercial de potência de motores, não é necessário aproximá-lo para outro 
valor mais próximo, acima da potência selecionada. A substituição do motor padrão pelo motor de alto rendimento proporcionará $2,3 \%$ de ganho no rendimento do sistema, pela aplicação da Eq. 3, para as condições preestabelecidas.

Tabela 3. Comparação entre a substituição de um motor padrão por outro motor de alto rendimento de mesma potência

\begin{tabular}{lc}
\multicolumn{1}{c}{ Parâmetros Avaliados (Motor Padrão) } & Valores \\
\hline Potência & $55,20 \mathrm{~kW}(75 \mathrm{cv})$ \\
Índice de carregamento & $80 \%$ \\
Rendimento & $91,80 \%$ \\
& Resultados (motor de alto rendimento) \\
Potência & $55,20 \mathrm{~kW}(75 \mathrm{cv})$ \\
Índice de carregamento & $80 \%$ \\
Rendimento do motor de alto rendimento & $93,80 \%$ \\
na condição de carga do motor padrão & \\
\hline
\end{tabular}

Com os dados de entrada e os índices econômicos dos motores padrão e de alto rendimento, pode-se fazer a análise econômica desses motores em estudo e concluir qual deles é economicamente viável para o horizonte de planejamento considerado. Ressalta-se que a diferença de custo observada entre os motores, deve ser compensada pela maior eficiência do uso de motores de alto rendimento, no horizonte de planejamento estudado; portanto, justifica-se a aquisição do motor de alto rendimento de mesma potência em situações onde o número de horas de funcionamento anual é relativamente elevado.

\section{Motor padrão em uso versus motor de alto rendimento novo, adequado à condição de carga}

A análise baseia-se nos parâmetros técnicos e econômicos considerados ao se efetuar a troca de um motor padrão em funcionamento, por outro motor de alto rendimento novo, adequado à nova condição de carregamento e potência. As Tabelas 4 e 5 exemplificam a metodologia utilizada para adequação da força motriz de um motor padrão em uso, por outro motor de alto rendimento novo adequado à condição de carga.

Como 44,20 kW (60 cv) é valor comercial de potência de motores, não é necessário ajustá-lo para o valor mais próximo de potência acima da selecionada. A potência demandada para realização do mesmo serviço foi reduzida de $55,20 \mathrm{~kW}$ (75 cv) para 44,20 kW (60 cv) proporcionando redução de $11 \mathrm{~kW}(15 \mathrm{cv})$ demandados, com uma economia de cerca de $2,30 \%$.

Como na escolha da potência mecânica requerida, a potência de 30,9 kW (42 cv) não é valor comercial de potência de motores, deve-se ajustá-la para o valor mais próximo da potência acima da selecionada; neste caso, o motor a ser adotado para um motor com potência igual a $36,80 \mathrm{~kW}(50 \mathrm{cv})$. O IC é calculado levando-se em consideração a potência do motor adequado ideal e o mais próximo vendido comercialmente. Então, o IC do motor seria $84 \%$, com um ganho de $6,50 \%$ no rendimento do sistema. A diferença de custo dos motores deve ser compensada pela maior eficiência do uso de motores de alto rendimento e da diminuição da potência requerida.
Tabela 4. Comparação entre a substituição de um motor padrão por um motor de alto rendimento adequado à carga

\begin{tabular}{lc}
\multicolumn{1}{c}{ Parâmetros Avaliados } & Valores \\
\hline Potência & $55,20 \mathrm{~kW}(75 \mathrm{cv})$ \\
Índice de carregamento & $80 \%$ \\
Rendimento & $91,50 \%$ \\
Resultados (motor - alto rendimento adequado à condição de carga) \\
Potência & $55,20 \times 0,80=44,2 \mathrm{~kW}(60 \mathrm{cv})$ \\
Índice de carregamento & $60 / 60=100 \%$ \\
Rendimento do motor de AR & $93,5 \%$ \\
\hline
\end{tabular}

Tabela 5. Comparação entre a substituição de um motor padrão em uso por um motor de alto rendimento adequado à condição de carga

\begin{tabular}{lc}
\hline \multicolumn{1}{c}{ Parâmetros Avaliados } & Valores \\
\hline Potência & $73,60 \mathrm{~kW}(100 \mathrm{cv})$ \\
Índice de carregamento & $42 \%$ \\
Rendimento & $87,50 \%$ \\
Resultados (motor - alto rendimento adequado a condição de carga) \\
Potência & $73,60 \times 0,42=30,90 \mathrm{~kW}(42 \mathrm{cv})$ \\
Como 30,90 kW (42 cv) não é & \\
valor comercial de potência de & $36,80 \mathrm{~kW}(50 \mathrm{cv})$ \\
motores - aproximar para o & \\
valor mais próximo de potência & \\
acima da selecionada & 42 / 50 $=84 \%$ \\
Índice de carregamento & $92,80 \%$ \\
Rendimento & \\
\hline
\end{tabular}

Motor padrão em uso, versus motor adequado à condição de carga, com número de horas de funcionamento anual maximizado

A Tabela 6 apresenta os resultados da aplicação da metodologia utilizada para substituição de um motor padrão por um motor de alto rendimento adequado à condição de carga, com o número de horas de funcionamento maximizado.

Nesta análise ocorreu a otimização do horário de funcionamento anual e, assim, considerou-se que o aumento no número de horas de funcionamento anual foi proporcional à diminuição da potência mecânica requerida. Como o aumento no número de horas de funcionamento anual foi de duas vezes, reduziu-se a potência à metade.

A potência mecânica requerida de $36,80 \mathrm{~kW}(50 \mathrm{cv})$ corresponde ao valor comercial de potência dos motores. Neste caso, o motor adotado foi o de potência igual a $36,80 \mathrm{~kW}(50 \mathrm{cv})$ com IC de $85 \%$ e um ganho de $1,20 \%$ no desempenho em relação ao motor padrão, mas com diminuição da potência mecânica requerida de $73,60 \mathrm{~kW}(50 \mathrm{cv})$ para $36,80 \mathrm{~kW}(50 \mathrm{cv})$. Nesta situação, uma análise da mão-de-obra deve ser feita pois, com a otimização do número de horas de funcionamento anual, poderá haver maior demanda de mão-de-obra.

É necessário um estudo detalhado sobre as implicações da maximização do número de horas de funcionamento anual, visto que o rendimento da carga não é, em geral, constante, podendo ocorrer aumento no consumo de energia elétrica. Estudos se fazem necessários para determinar se os ganhos com a diminuição da demanda compensarão o possível aumento no consumo de energia elétrica e de mão-de-obra (Campana, 2000). O possível aumento no consumo de energia elétrica se deve ao fato de que motores menores, que provavelmente serão demandados, são mais ineficientes que os maiores. 
Tabela 6. Comparação entre a substituição de um motor padrão em uso por um motor de alto rendimento adequado à condição de carga com número de horas de funcionamento anual maximizado

\begin{tabular}{ll}
\multicolumn{1}{c}{ Parâmetros Avaliados } & \multicolumn{1}{c}{ Valores } \\
\hline Potência & $73,60 \mathrm{~kW}(100 \mathrm{cv})$ \\
Índice de carregamento & $85 \%$ \\
Número de horas de funcionamento anual & $1.500 \mathrm{~h}$ \\
Rendimento & $92,20 \%$ \\
\multicolumn{2}{c}{ Resultados (motor de alto rendimento adequado e otimizado) } \\
Número de horas de funcionamento anual & $3.000 \mathrm{~h}$ \\
Potência & $73,60(1.500 / 3.000)=$ \\
Motor escolhido & $36,80 \mathrm{~kW}(50 \mathrm{cv})$ \\
Índice de carregamento & $50 \mathrm{cv}$ \\
Rendimento & $85 \%$ \\
\hline
\end{tabular}

A Tabela 7 apresenta informações características dos motores elétricos em condição de funcionamento diário, na fábrica de ração e nela estão descritas as condições de trabalho dos motores e algumas características, como o tipo de motor, rotação nominal, potência e horário de funcionamento diário, dentre outros.

A Tabela 8 apresenta os índices econômicos utilizados para realização da análise econômica. Na Tabela 8 são descritas as informações necessárias para realização da análise econômica dos motores elétricos, para melhor opção de troca, tais como preço do motor, taxa de atratividade de investimento, número de horas de funcionamento anual da fábrica de ração, rendimento e índice de carregamento em plena carga, dentre outras.

Com os dados das Tabelas 7 e 8 , foi possível realizar-se a análise econômica dos motores elétricos para a situação mais vantajosa de investimento. A Tabela 9 (A, B, C e D) apresentam o resumo da análise econômica para a melhor opção de investimento, dentre as estudadas para os motores do moinho, misturador, exaustor e sistema de transporte, respectivamente.

De acordo com a Tabela 9A, a melhor opção de troca de motores para o moinho, dentre as opções estudadas, é a substituição do motor padrão atual de $36,80 \mathrm{~kW}(50 \mathrm{cv})$ com IC de $106 \%$, por um motor de alto rendimento com potência de $18,40 \mathrm{~kW}(25 \mathrm{cv})$ adequado à condição de carga, com o número de horas de funcionamento anual maximizado, com IC de 106\%; logo, a economia anual será de $22,90 \%$. Os parâmetros econômicos VPL de R \$10.134,90, TIR que não converge, RBC de 17,9 e TRC imediato reforçam a tomada de decisão.

Segundo a Tabela 9B, dentre as alternativas estudadas a melhor opção de troca para o misturador seria a substituição do motor padrão de $14,70 \mathrm{~kW}(20 \mathrm{cv})$ com IC de $48 \%$, por um motor de alto rendimento adequado à condição de carga de $5,50 \mathrm{~kW}$ (7,5 cv) com IC de 97,30\%; então, a economia anual seria de 23,50\%. Parâmetros econômicos como VPL de R \$2.437,70, TIR que não converge, RBC de 4,70 e TRC imediato, reforçam a tomada de decisão por um motor de alto rendimento, adequado à condição de carga de 5,50 kW (7,5 cv).

Tabela 7. Levantamento de dados dos motores elétricos da fábrica de ração da UFV

\begin{tabular}{|c|c|c|c|c|}
\hline Equipamento & Moinho Principal & Misturador & Exaustor & Sistema de Transporte \\
\hline Fabricante & WEG & ARNO & WEG & ARNO \\
\hline Número de pólos & 2 pólos & 6 pólos & 2 pólos & 4 pólos \\
\hline Freqüência (Hz) & 60 & 60 & 60 & 60 \\
\hline Potência $(\mathrm{kW})$ & $36,80(50 \mathrm{cv})$ & $14,70(20 \mathrm{cv})$ & $11,00(15 \mathrm{cv})$ & $5,50(7,5 \mathrm{cv})$ \\
\hline Corrente nominal (A) & $125 / 72$ & $58 / 33,50$ & $38 / 22$ & $21,5 / 12,40$ \\
\hline Tensão nominal (V) & $220 / 380$ & $220 / 380$ & $220 / 380$ & $220 / 380$ \\
\hline Rendimento nominal (\%) & 92,20 & 89,00 & 89,50 & 88,50 \\
\hline Número de fases & 3 & 3 & 3 & 3 \\
\hline Motor de indução tipo gaiola ou curto circuitado & Indução & Indução & Indução & Indução \\
\hline Rotação (rpm) & 3.560 & 880 & 3.500 & 1.730 \\
\hline Tipo de ligação do motor & Delta & Delta & Delta & Delta \\
\hline Padrão ou Alto rendimento & Padrão & Padrão & Padrão & Padrão \\
\hline Horário de funcionamento fora de ponta/dia (h) & 8 & 8 & 8 & 8 \\
\hline Corrente em cada fase e sob carga (A) & $125 / 130 / 125$ & $38 / 39,50 / 39$ & $20 /-/-$ & $9,20 / 8,90 / 10,40$ \\
\hline Forma de acoplamento & Direto & Polia / Correia & Polia / Correia & Polia / Correia \\
\hline Vibração no motor & Não & Não & Não & Não \\
\hline
\end{tabular}

Tabela 8. Índices utilizados na análise econômica

\begin{tabular}{|c|c|c|c|c|c|}
\hline Motor & Unidade & \multicolumn{4}{|c|}{ Motor Padrão ou Alto Rendimento } \\
\hline Potência & $\mathrm{kW}$ & $36,80(50 \mathrm{cv})$ & $14,70(20 \mathrm{cv})$ & $11,00(15 \mathrm{cv})$ & $5,50(7,5 \mathrm{cv})$ \\
\hline Índice de carregamento & $\%$ & 106 & 48 & 42 & 10 \\
\hline Rendimento & $\%$ & 91,80 & 86,20 & 84,90 & 63,10 \\
\hline Preço do motor & $\mathrm{R} \$$ & $2.152,60$ & $1.226,20$ & 639,50 & 445,10 \\
\hline Número de horas de funcionamento anual & $\mathrm{h}$ & \multicolumn{4}{|c|}{1.500} \\
\hline Número de horas de funcionamento anual maximizado & $\mathrm{h}$ & \multicolumn{4}{|c|}{3.000} \\
\hline Juros (custo de oportunidade) & $\% /$ ano & \multicolumn{4}{|c|}{8} \\
\hline Valor de sucata no ano final da vida útil em relação a um motor novo & $\%$ & \multicolumn{4}{|c|}{20} \\
\hline Custo unitário da energia fora de ponta & $\mathrm{R} \$ \mathrm{kWh}^{-1}$ & \multicolumn{4}{|c|}{0,05} \\
\hline Custo unitário da demanda fora de ponta & $\mathrm{R} \$ \mathrm{~kW}^{-1}$ & \multicolumn{4}{|c|}{4,90} \\
\hline Aumento do preço da energia acima da inflação & $\% /$ ano & \multicolumn{4}{|c|}{2} \\
\hline Manutenção & $\%$ & \multicolumn{4}{|c|}{1} \\
\hline Horizonte de planejamento & ano & \multicolumn{4}{|c|}{10} \\
\hline
\end{tabular}


Tabela 9. Análise econômica da adequação de força motriz para o motor do moinho principal; Misturador, exaustor e sistema de transporte*

\begin{tabular}{|c|c|c|c|c|c|c|c|}
\hline \multicolumn{2}{|l|}{ Dados } & Motor PD & Motor AR AD/h & \multicolumn{4}{|c|}{ Motor PD versus Motor AR AD/h } \\
\hline \multicolumn{8}{|l|}{ A. Moinho Principal } \\
\hline Consumo de energia elétrica & $\mathrm{kWh} \mathrm{ano}^{-1}$ & $63.489,60$ & $63.263,10$ & Potência & $\mathrm{kW}(\mathrm{cv})$ & $36,80(50)$ & $18,40(25)$ \\
\hline Conta de energia & $\mathrm{R} \$ \mathrm{ano}^{-1}$ & $5.628,80$ & $4.341,10$ & Carga & $\%$ & 106 & 106 \\
\hline Preço do motor & $\mathrm{R} \$$ & $2.152,60$ & $1.586,40$ & Rendimento & $\%$ & 91,80 & 92,50 \\
\hline \multicolumn{4}{|c|}{ Economia anual com energia elétrica (R\$) } & \multicolumn{2}{|c|}{$1.287,70(22,9 \%)$} & & \\
\hline Análise econômica & $P D-P D A D$ & \multicolumn{2}{|r|}{$\mathrm{PD}-\mathrm{AR}$} & \multicolumn{2}{|c|}{$\mathrm{PD}-\mathrm{AR} A D$} & \multicolumn{2}{|c|}{$\mathrm{PD}-\mathrm{AR} \mathrm{AD} / \mathrm{h}$} \\
\hline VPL (R\$) & - & \multicolumn{2}{|r|}{$-344,80$} & \multicolumn{2}{|c|}{$-344,80$} & \multicolumn{2}{|c|}{$10.134,90$} \\
\hline $\operatorname{TIR}(\%)$ & - & \multicolumn{2}{|r|}{-7} & \multicolumn{2}{|c|}{-7} & \multicolumn{2}{|c|}{ Não converge } \\
\hline $\mathrm{RBC}$ & - & \multicolumn{2}{|c|}{0,6} & \multicolumn{2}{|c|}{0,6} & \multicolumn{2}{|c|}{17,9} \\
\hline TRC (ano) & - & \multicolumn{2}{|c|}{ Não retornável } & \multicolumn{2}{|c|}{ Não retornável } & \multicolumn{2}{|c|}{ Imediato } \\
\hline \multicolumn{8}{|l|}{ B. Misturador* } \\
\hline Preço do motor & $\mathrm{R} \$$ & $1.136,20$ & 850,60 & Rendimento & & 86,20 & 87,50 \\
\hline \multicolumn{2}{|c|}{ Economia anual com energia elétrica $(\mathrm{R} \$)$} & \multirow{2}{*}{\multicolumn{2}{|c|}{$\mathrm{AR}$}} & \multicolumn{2}{|c|}{$255,12(23,5 \%)$} & & \\
\hline Análise econômica $\quad$ PD & $\mathrm{PD} A D$ & & & $\mathrm{AR} A D$ & & \multicolumn{2}{|c|}{$\mathrm{AR} A D h^{-1}$} \\
\hline $\mathrm{VP}\left(\mathrm{R} \$ \mathrm{MJ}^{-1}\right) \quad 0,0211$ & 0,0197 & & 0228 & 0,0208 & & 0,015 & \\
\hline Análise econômica & $\mathrm{PD}-\mathrm{PD} \mathrm{AD}$ & $\mathrm{PD}$ & $-\mathrm{AR}$ & $\mathrm{PD}-\mathrm{AR} A D$ & & $\mathrm{PD}-\mathrm{AR} A$ & $h^{-1}$ \\
\hline VPL $(\mathrm{R} \$)$ & 504,50 & & 33,00 & 548,40 & & 2.437 & \\
\hline TIR $(\%)$ & - & & -22 & Não converge & & Não conv & rge \\
\hline $\mathrm{RBC}$ & 1,0 & & 0,9 & 3,0 & & 4,7 & \\
\hline TRC (ano) & Imediato & Não re & etornável & Imediato & & Imedia & \\
\hline
\end{tabular}

\section{Exaustor*}

Consumo de energia elétrica

Demanda de energia elétrica

Conta de energia

Preço do motor

Economia anual com energia elétrica $(\mathrm{R} \$)$

$\begin{array}{cc}\text { Análise econômica } & \text { PD } \\ \operatorname{VP}\left(\mathrm{R} \$ \mathrm{MJ}^{-1}\right) & 0,0206\end{array}$

Análise econômica

VPL (R\$)

TIR (\%)

$\mathrm{RBC}$

TRC (ano)

$\begin{array}{ccc}\mathrm{kWh}_{\mathrm{ano}}{ }^{-1} & 8.192 .20 & 8.809,50 \\ \mathrm{~kW} \text { ano }^{-1} & 65,5 & 34,4 \\ \mathrm{R} \$ \mathrm{ano}^{-1} & 723,7 & 590,5 \\ \mathrm{R} \$ & 592,5 & 342,3\end{array}$

PD ADQ

0,0197

PD - PD AD

371,60

Não converge

1,5

Imediato

$\mathrm{AR}$
0,0217
$\mathrm{PD}-\mathrm{AR}$
95,00
1
0,3
Imediato

$\begin{array}{cccc}\text { Potência } & \mathrm{kW}(\mathrm{cv}) & 11,00(15) & 2,90(4) \\ \text { Horas anuais } & \mathrm{h} / \text { ano } & 1.500 & 3.000 \\ \text { Carga } & \% & 42 & 84 \\ \text { Rendimento } & \% & 84,90 & 86,20\end{array}$

$$
\begin{gathered}
133,3(18,4 \%) \\
\text { AR ADQ } \\
0,0203 \\
\text { PD - AR AD } \\
240,00
\end{gathered}
$$

Não converge

$$
4,3
$$

\begin{tabular}{|c|c|c|c|c|c|c|c|}
\hline Consumo de energia elétrica & $\mathrm{kWh}$ ano $^{-1}$ & $2.345,9$ & 2259,5 & Potência & $\mathrm{kW}(\mathrm{cv})$ & $5,52(7,5)$ & $1,47(2)$ \\
\hline Demanda de energia elétrica & $\mathrm{kW}$ ano $^{-1}$ & 18,8 & 9,04 & Horas anuais & $\mathrm{h} / \mathrm{ano}$ & 1.500 & 1.500 \\
\hline Conta de energia & $\mathrm{R} \$ \mathrm{ano}^{-1}$ & 115,9 & 155,1 & Carga & $\%$ & 22 & 82,50 \\
\hline Preço do motor & $\mathrm{R} \$$ & 207,7 & 144,3 & Rendimento & $\%$ & 77,7 & 80,60 \\
\hline \multicolumn{4}{|c|}{ Economia anual com energia elétrica (R\$) } & \multicolumn{4}{|c|}{$52,2(25,2 \%)$} \\
\hline Análise econômica $\quad$ PD & \multicolumn{2}{|c|}{ PD ADQ } & AR & \multicolumn{2}{|c|}{ AR ADQ } & \multicolumn{2}{|c|}{ AR ADQ/h } \\
\hline $\mathrm{VP}\left(\mathrm{R} \$ \mathrm{MJ}^{-1}\right)$ & \multicolumn{2}{|c|}{0,0207} & 0,0238 & \multicolumn{2}{|c|}{0,0219} & \multicolumn{2}{|c|}{0,0160} \\
\hline Análise econômica & \multicolumn{2}{|c|}{ PD-PD ADQ } & $P D-A R P$ & \multicolumn{2}{|c|}{$\mathrm{PD}-\mathrm{AR} A \mathrm{ADQ}$} & \multicolumn{2}{|c|}{$\mathrm{PD}-\mathrm{AR} \mathrm{ADQ} / \mathrm{h}$} \\
\hline VPL $(\mathrm{R} \$)$ & \multicolumn{2}{|c|}{290,8} & 6,50 & \multicolumn{2}{|c|}{286,20} & \multicolumn{2}{|c|}{707,30} \\
\hline $\operatorname{TIR}(\%)$ & \multicolumn{2}{|c|}{ Não converge } & - & \multicolumn{2}{|c|}{ Não converge } & \multicolumn{2}{|c|}{ Não converge } \\
\hline $\mathrm{RBC}$ & \multicolumn{2}{|c|}{1,17} & - & \multicolumn{2}{|c|}{1,67} & \multicolumn{2}{|c|}{2,35} \\
\hline TRC (ano) & \multicolumn{2}{|c|}{ Imediato } & Imediato & \multicolumn{2}{|c|}{ Imediato } & \multicolumn{2}{|c|}{ Imediato } \\
\hline
\end{tabular}

9

$$
\begin{gathered}
\mathrm{AR} A D Q / h \\
0,0153 \\
\mathrm{PD}-\mathrm{AR} \mathrm{AD} / \mathrm{h} \\
1.274,40
\end{gathered}
$$

Não converge 4,7

D. Sistema de transporte* 
De acordo com a Tabela 9C, a melhor opção de troca para o motor do exaustor, dentre as possíveis estudadas, é a substituição do motor padrão atual de $11,0 \mathrm{~kW}(15 \mathrm{cv})$ com IC de $42 \%$, por um motor de alto rendimento adequado à condição de carga, com o número de horas de funcionamento anual maximizado de $2,9 \mathrm{~kW}$ (4 cv), com IC de 84\%; assim, a economia anual é de 18,4\%. Parâmetros econômicos como VPL de R \$ 1.274,4; TIR não-convergente; RBC de 4,7 e TRC imediato, reforçam a tomada de decisão por um motor de alto rendimento de $2,9 \mathrm{~kW}(4 \mathrm{cv})$ adequado à condição de carga, com o número de horas de funcionamento anual maximizado.

De acordo com a Tabela 9D, a melhor opção de troca para o motor do sistema de transporte, dentre as alternativas estudadas, seria a substituição do motor padrão atual de 5,50 $\mathrm{kW}(7,5 \mathrm{cv})$ com IC de $22 \%$, por um motor de alto rendimento adequado à condição de carga, com o número de horas de funcionamento anual maximizado de $1,47 \mathrm{~kW}(2 \mathrm{cv})$ e IC de $80,60 \%$; desta forma, a economia anual seria de $25,20 \%$. Os parâmetros econômicos VPL de R \$ 707,32, TIR não-convergente, RBC de 2,4 e TRC imediato, reforçam a tomada de decisão.

$\mathrm{O}$ potencial total de economia de energia elétrica anual estimado foi de $\mathrm{R} \$ 1.728,30(22,60 \%)$ caso se faça o aumento do número de horas de funcionamento anual e a adequação de força motriz com o uso de motores de alto rendimento.

O estudo detalhado de adequação de força motriz deverá contemplar, também, a adequação do sistema de comando e proteção e o custo de instalação de motores elétricos, que não foram considerados neste trabalho.

\section{CONCLUSÕES}

1. A metodologia de adequação de força motriz mostrou-se eficaz e de fácil manuseio.

2. O potencial de redução nos gastos com energia elétrica, na fábrica de ração, por meio da adequação de força motriz, foi de $22,60 \%$

3. As particularidades do processo, tais como as exigências eletromecânicas do moinho e o horário de funcionamento da fábrica de ração, permitem efetivar-se a substituição por motores adequados e mais eficientes

4. Motores adequados e eficientes realizam o mesmo trabalho dos motores em funcionamento, demandando menor potência e gastos com energia elétrica

5. Pode-se racionalizar o custo operacional e o uso da energia elétrica trocando-se o motor padrão de $36,80 \mathrm{~kW}(50 \mathrm{cv})$ por outro de alto rendimento, com potência de $17,40 \mathrm{~kW}(25 \mathrm{cv})$, alterando-se o número de horas de funcionamento anual de 1.500 horas para 3.000 horas, sem alterar a produção de ração diária. A alteração no número de horas de operação da fábrica implica em um estudo do impacto do custo de mão-de-obra e encargos sociais, para a tomada de decisão
6. Há a necessidade da certificação dos equipamentos de transporte utilizados em fábricas de ração.

\section{LITERATURA CITADA}

Campana, S. Racionalização do uso de energia elétrica em sistemas de irrigação tipo pivô central e aspersão convencional. Viçosa, MG: UFV, Imprensa Universitária, 2.000. 108p. Dissertação Mestrado

CASP S.A. Indústria e Comércio; Manual - fábrica de ração. CASP, 1980. 18p.

Costa, J.M.; Oliveira Filho; D.; Teixeira, C.A. Adequação de força motriz - Estudo de caso na fabricação de papel reciclado. In: Congresso Latino-Americano de Distribuição de Energia Elétrica - CONLADIS, 3, 1998. São Paulo. Resumos... São Paulo: Universidade de São Paulo, 1998. p. 561-564.

ELETROBRÁS - Guia operacional de motores elétricos. In: Programa de Combate ao Desperdício de Energia Elétrica PROCEL. Rio de Janeiro: PROCEL, 1998. v.1, 161p.

Jannuzzi, G.M.; Swisher, J.N.P. Planejamento integrado dos recursos energéticos: meio ambiente, conservação de energia e fontes renováveis. 1 ed. Campinas, 1997. 246p.

Latorre, C. de F.; Nobre, E.C.; Burgoa, J.A. Diagnóstico do potencial de conservação de energia na indústria. In: Seminário Internacional de Distribuição de Energia Elétrica, 1, 1990. Belo Horizonte. Resumos... Belo Horizonte: Companhia Energética de Minas Gerais, 1990. v.1. 3. 5p.

Low, S. 1992 Industrial sector end-use forecast. Toronto: Ontario Hydro, 1993. 44p. Main Report

Pinheiro, S.F. Conservação de energia elétrica: recurso energético planificado. In: Congresso Brasileiro de Planejamento Energético, 1, Rio de Janeiro. Anais... Rio de Janeiro: v.l. 3, p. 95-108. 1986

Sá, J.S.; Cogo, J.R.; Arango, H. Avaliação do rendimento de motores de indução trifásicos via medição de tensão e corren-te no estator. In: Seminário Internacional de Distribuição de Energia Elétrica, 1, 1990. Belo Horizonte. Resumos... Belo Horizonte: Companhia Energética de Minas Gerais, 1990. v.l. 3.4p

Teixeira, C.A. Metodologia para adequação do uso de força motriz em processos agrícolas. Viçosa, MG: UFV, Imprensa Universitária, 2.002. 155p. Dissertação Mestrado.

WEG, S.A. Catálogo eletrônico 2000. Jaraguá do Sul, RS. (http: //www. weg.com.br), Data da consulta: 01/07/2001.

Zanotto, D.; Monticelli, C.; Mazzuco, H. Implicações da granulometria de ingredientes de rações sobre a produção de suínos e aves. In: Simpósio Latino-Americano de Nutrição de Suínos e Aves, 1995. Campinas. Anais... Campinas: Colégio Brasileiro de Nutrição Animal (CBNA), 1995.p.111-134. 